\title{
温和条件下去丙酮保护制备末端单炔烃及末端二炔的方法
}

\author{
关志朋师瑶石炜*陈浩 \\ (华中农业大学理学院 武汉 430070)
}

\begin{abstract}
摘要 末端炔烃是有机合成中重要的原料或中间体. 通过钯催化的 Sonogashira 偶联反应，将 2-甲基-3-丁炔-2-醇连接 到其它基团上所得到的结构，可以看作是一类丙酮保护的末端炔烃. 目前，对于这种丙酮保护的末端炔烃，其去保护 得到端基炔主要是通过 $\mathrm{KOH}$ 存在下在甲苯中回流数小时的方法, 但是反应温度高, 条件苛刻, 适用范围较窄. 对这一 脱保护过程进行研究, 通过对多种溶剂和反应温度进行对比, 发现以 1,4-二氧六环作为溶剂, KOH 作为碱, 可以在相对 较低的温度 $\left(60{ }^{\circ} \mathrm{C}\right)$ 下得到较高的产率, 而且所需时间大大缩短 $(0.5 \sim 2 \mathrm{~h})$, 拓展了这种方法的应用范围.
\end{abstract}

关键词 1,4-二氧六环; 去保护; 末端炔烃; 2-甲基-3-丁炔-2-醇

\section{Synthesis of Terminal Alkynes/Diynes through Deprotection of Acetone Protected Alkynes under Mild Conditions}

\author{
Guan, Zhipeng Shi, Yao Shi, Wei* Chen, Hao \\ (College of Science, Huazhong Agricultural University, Wuhan 430070)
}

\begin{abstract}
Terminal alkynes are important building blocks in organic synthesis. Acetone protected terminal alkynes are frequently used in alkyne synthesis and reactions, which could be easily prepared by Pd-catalyzed Sonogashira cross-coupling reactions between electrophiles and 2-methyl-3-butyn-2-ol. At present, the deprotection of the acetone protected terminal alkynes is generally carried out in reflux toluene in the presence of potassium hydroxide for hours. However, this procedure suffers from the harsh reaction conditions and its application has been limited. Here in the deprotection of acetones has been discussed, and various solvent, temperature, substrates were investigated to advance the procedure, and it is found that by employing 1,4-dioxane as solvent, this process can undergo smoothly at $60{ }^{\circ} \mathrm{C}$ with satisfying yields in $0.5 \sim 2 \mathrm{~h}$ in the presence of 2.0 equiv. $\mathrm{KOH}$.
\end{abstract}

Keywords 1,4-dioxane; deprotection; terminal alkyne; 2-methyl-3-butyn-2-ol

具有芳基末端炔烃结构的化合物, 不仅是有机合成 中重要的原料或者中间体, 广泛应用于农药以及药物等 合成过程中, 而且还是许多天然产物和具有生理活性的 化合物的核心骨架 ${ }^{[1 \sim 3]}$, 还可以通过末端炔烃之间的二 聚反应得到共轭多炔结构 ${ }^{[4 \sim 7]}$, 因此发展这类炔烃具有 重要的意义和广阔的应用前景 ${ }^{[8 \sim 10]}$. 目前, 合成端基炔 化合物的过程, 一种重要的方法是通过卤代烃和 2-甲 基-3-丁炔-2-醇的 Sonogashira 偶联 ${ }^{[11 ~ 13]}$, 得到的产物可 以看作是丙酮保护的末端炔烃 ${ }^{[14]}$. 该产物在一定条件 下脱去丙酮的保护, 即得炔烃, 如图 1 所示.

如果将图 1 中的 2-甲基-3-丁炔-2-醇替换成乙炔或
三甲基硅基乙炔 ${ }^{[15]}$, 反应也可以进行. 但是，乙炔难以 操作、储存、运输，且使用过程中有一定的危险性; 三 甲基硅基乙炔虽然反应迅速、脱保护条件温和 ${ }^{[16]}$, 但是 价格却非常昂贵 ${ }^{[17]}$. 相比较而言, 2-甲基-3-丁炔-2-醇的 价格低廉 ${ }^{177}$, 易于存储和运输, 反应简便而迅速, 具备 更好的应用前景, 但是存在的问题是其脱保护操作较困 难. 根据已有报道, 2-甲基-3-丁炔-2-醇需要在 $\mathrm{KOH}$ 、 $\mathrm{NaOH}$ 或 $\mathrm{NaH}$ 等强碱存在下, 甲苯中 $110{ }^{\circ} \mathrm{C}$ 回流数小时 才能脱去丙酮的保护 ${ }^{[14,18,19]}$, 部分文献曾报道在 $70{ }^{\circ} \mathrm{C}$ 下可以得到部分炔烃产物 ${ }^{[20,21]}$, 但产率较低(58\%), 因 此限制了其应用. Huang 等 ${ }^{[22]}$ 发现在甲醇-甲苯混合溶

* Corresponding author. E-mail: shiwei@mail.hzau.edu.cn

Received July 20, 2016; revised September 19, 2016; published online October 11, 2016.

Project supported by the National Natural Science Foundation of China (No. 21202050), and the Natural Science Foundation of Hubei Province (No. 2014CFB930).

国家自然科学基金(No. 21202050)、湖北省自然科学基金(No. 2014CFB930)资助项目. 


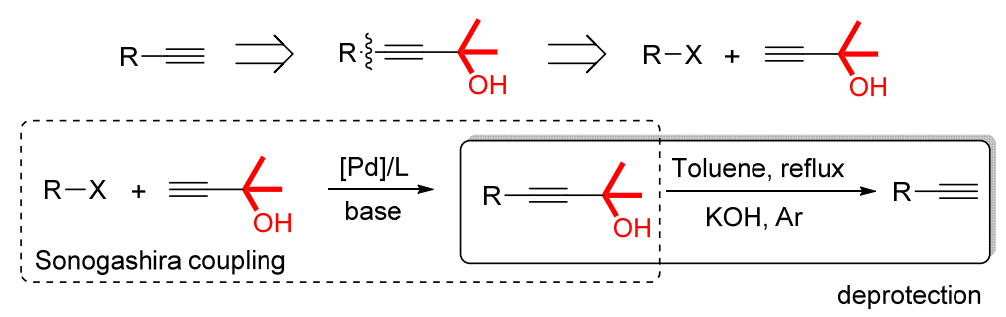

图 1 Sonogashira 偶联制备端炔的方法

Figure 1 Sonogashira coupling to prepare terminal alkynes

剂中, $75{ }^{\circ} \mathrm{C}$ 下四丁基氢氧化铵也可以得到炔烃, 但是四 丁基氢氧化铵价格昂贵, 且对制备、运输、操作等环节 都有较高的要求. $\mathrm{Li}$ 等 ${ }^{[23]}$ 则发现使用二乙二醇单乙基醚 和甲苯作为混合溶剂, $\mathrm{NaOH}$ 为碱, $75{ }^{\circ} \mathrm{C}$ 下可以得到末 端炔烃, 但是需要 5 倍量以上的强碱, 且在报道的反应 中, 产率均未超过 $90 \%$. 本研究针对这一问题, 对丙酮 保护的末端炔烃的去保护进行了研究, 发现可以在更低 的温度下, 在较短时间内 $(0.5 \sim 2 \mathrm{~h})$ 即可实现这一去保 护过程, 且反应体系更简便, 实现了末端 1,3-二炔及末 端炔烃的相对简便和廉价的合成, 具备一定的有机合成 意义.

\section{1 结果与讨论}

\section{1 合成丙酮保护的末端炔烃}

按照 Eq. 1 所示, 合成丙酮保护的末端炔烃 ${ }^{[18]}$, 结 果如表 1 所示.

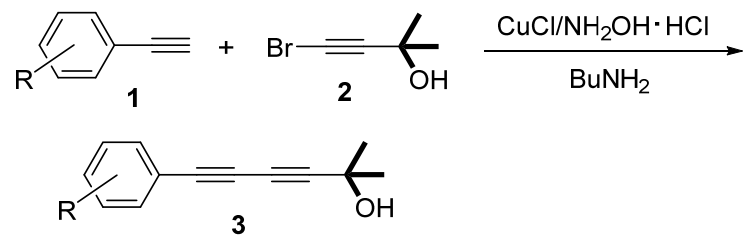

表 1 合成丙酮保护的末端 1,3-二炔烃

Table 1 Synthesis of acetone protected 1,3-butadiynes

\begin{tabular}{clcc}
\hline Entry & $\mathrm{R}$ & $\mathbf{3}$ & Isolated yield $/ \%$ \\
\hline 1 & $\mathrm{H}$ & $\mathbf{3 a}$ & 91 \\
2 & $4-\mathrm{F}$ & $\mathbf{3 b}$ & 88 \\
3 & $4-\mathrm{OEt}$ & $\mathbf{3 c}$ & 92 \\
4 & $4-\mathrm{Me}$ & $\mathbf{3 d}$ & 86 \\
\hline
\end{tabular}

以 $3 \mathbf{a}$ 的合成为例, 量取 $28 \mathrm{~mL}$ 正丁胺于 $150 \mathrm{~mL}$ 烧 瓶中, 加入 $43.56 \mathrm{mg}(0.44 \mathrm{mmol}) \mathrm{CuCl}$, 再分 2 4 次加 入过量的盐酸差弪胺 (约 $0.5 \sim 0.8 \mathrm{mmol}$ ), 使体系保持为还 原态. 加入 $2.04 \mathrm{~g}(20 \mathrm{mmol})$ 苯乙炔, $0{ }^{\circ} \mathrm{C}$ 下, 边搅拌边

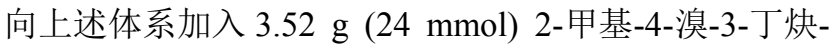
2-醇(2)反应 $5 \mathrm{~h}, \mathrm{TLC}$ 检测反应结束后, 以乙醚萃取分 离, 无水硫酸钠干燥后, 减压蒸发除去溶剂, 重结晶提 纯得到 $3.33 \mathrm{~g} \mathrm{3a}$, 产率 $91 \%$.

\section{2 合成末端炔烃标样}

参考传统方法 ${ }^{[14]}$, 将 $233 \mathrm{mg}(1.27 \mathrm{mmol})$ 原料 3a 溶 于经金属钠回流处理过的干甲苯 $(25 \mathrm{~mL})$ 中，加入 $80 \mathrm{mg}$ $\mathrm{NaOH}$, 氩气保护下回流, 薄层色谱(TLC)检测至反应完 全. 石油醚作流动相进行硅胶柱色谱分离提纯，最终得 到端基炔化合物 $\mathbf{4 a}$ (产率 $70 \%$ ), 为浅黄色油状液体，留 作气相色谱(GC)标准样品, 如 Eq. 2 所示.

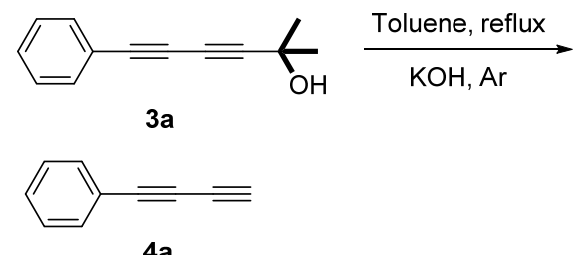

\section{3 脱保护反应条件优化}

分别取 $92 \mathrm{mg}(0.5 \mathrm{mmol}) 3 \mathrm{a}$ 溶于 $2 \mathrm{~mL}$ 甲苯、甲醇、 乙醇、二氯甲烷、四氢呋喃、1,4-二氧六环、甲基叔丁 基醚七种溶剂中, 加入 $56 \mathrm{mg}(1.0 \mathrm{mmol}) \mathrm{KOH}, 70{ }^{\circ} \mathrm{C}$ 氩 气保护下反应 $2 \mathrm{~h}$, 以联苯作为内标进行气相色谱分析. $\mathrm{GC}$ 产率如表 2 所示. 可以看出 1,4-二氧六环条件下有较 高的产率.

表 2 条件篮选

Table 2 Screening of reaction conditions

\begin{tabular}{clcc}
\hline Entry & \multicolumn{1}{c}{ 溶剂 } & $t /{ }^{\circ} \mathrm{C}$ & $\mathrm{GC}$ 产率 $/ \%$ \\
\hline 1 & 甲苯 & 70 & 42 \\
2 & 甲醇 & 70 & 7 \\
3 & 乙醇 & 70 & 16 \\
4 & 二氯甲烷 & 70 & 58 \\
5 & 四氢呋喃 & 70 & 40 \\
6 & $1,4-二$ 氧六环 & 70 & 74 \\
7 & 甲基叔丁基醚 & 70 & 44 \\
8 & 1,4-二氧六环 & 50 & 53 \\
9 & $1,4-二$ 氧六环 & 60 & 88 \\
\hline
\end{tabular}

随后以 1,4-二氧六环为溶剂, 对反应温度进行了探 索，发现 $60{ }^{\circ} \mathrm{C}$ 时反应的产率达到 $88 \%$ ，低于或高于此 温度, 反应产率均有所下降. 因此, 我们确定了最佳的 反应条件: 2 倍量的 $\mathrm{KOH}$ 存在条件下, 以 1,4-二氧六环 为溶剂, $60{ }^{\circ} \mathrm{C}$ 下反应. 
对反应时间也进行了研究, 结果如图 2 所示. 我们 发现此反应在 $15 \mathrm{~min}$ 内可基本完成, $30 \mathrm{~min}$ 左右达到最 大收率. 随着反应时间的延长, 产率反而有下降的趋势. 通过 GC-MS 及 NMR 等手段进行分析, 发现在数小时 后, 有 $20 \%$ 左右的产物转化为结构相对比较复杂的混合 物, 其结构包含炔基、甲基等, 但是通过薄层层析对比, 并未发现有末端炔烃自身偶联的四炔产物. 进一步的研 究目前正在进行中.

\section{4 底物的扩展}

按照文献方法, 合成了包含不同取代基的底物 3, 包括含二炔结构和含单炔结构的底物, 并对其进行脱保 护试验, 结果如表 3 所示. 可以看到, 不论是富电子取 代基还是贫电子取代基, 均可以满意的产率得到末端炔 烃的产物. 对于产物为共轭二炔的反应, 基本上可以在 $30 \mathrm{~min}$ 之内完成(Entries 1 5); 而对于产物为单炔的反 应，反应时间需延长到 $2 \mathrm{~h}$, 但温度保持不变(Entries 6 9). 对于 Entry 6, $\mathrm{GC}$ 显示反应物 $\mathbf{3 f}$ 基本完全转化, 但由 于产物苯乙炔沸点较低, 因此对其分离产率造成了影

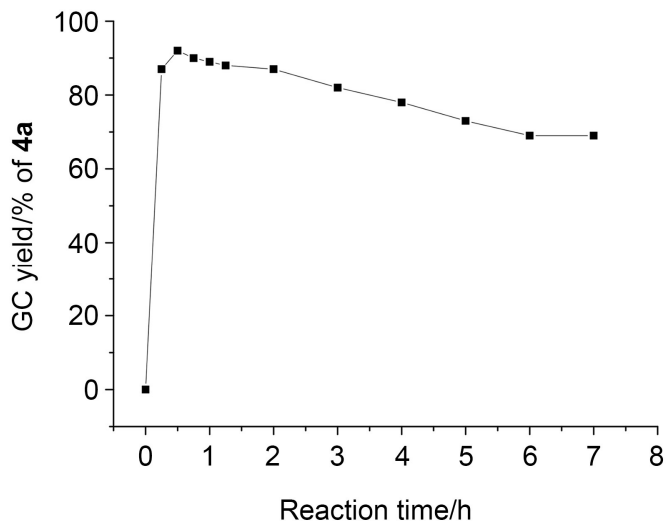

图 2 反应时间的影响

Figure 2 Optimization of reaction time 响.

\section{2 结论}

研究了丙酮保护的末端炔烃及二炔烃在温和条件 下去保护的方法, 发现该方法可以实现较低的反应温度 $\left(60{ }^{\circ} \mathrm{C}\right)$ 、较短的反应时间 $(30 \mathrm{~min} \sim 2 \mathrm{~h})$ 、较高的收率, 相

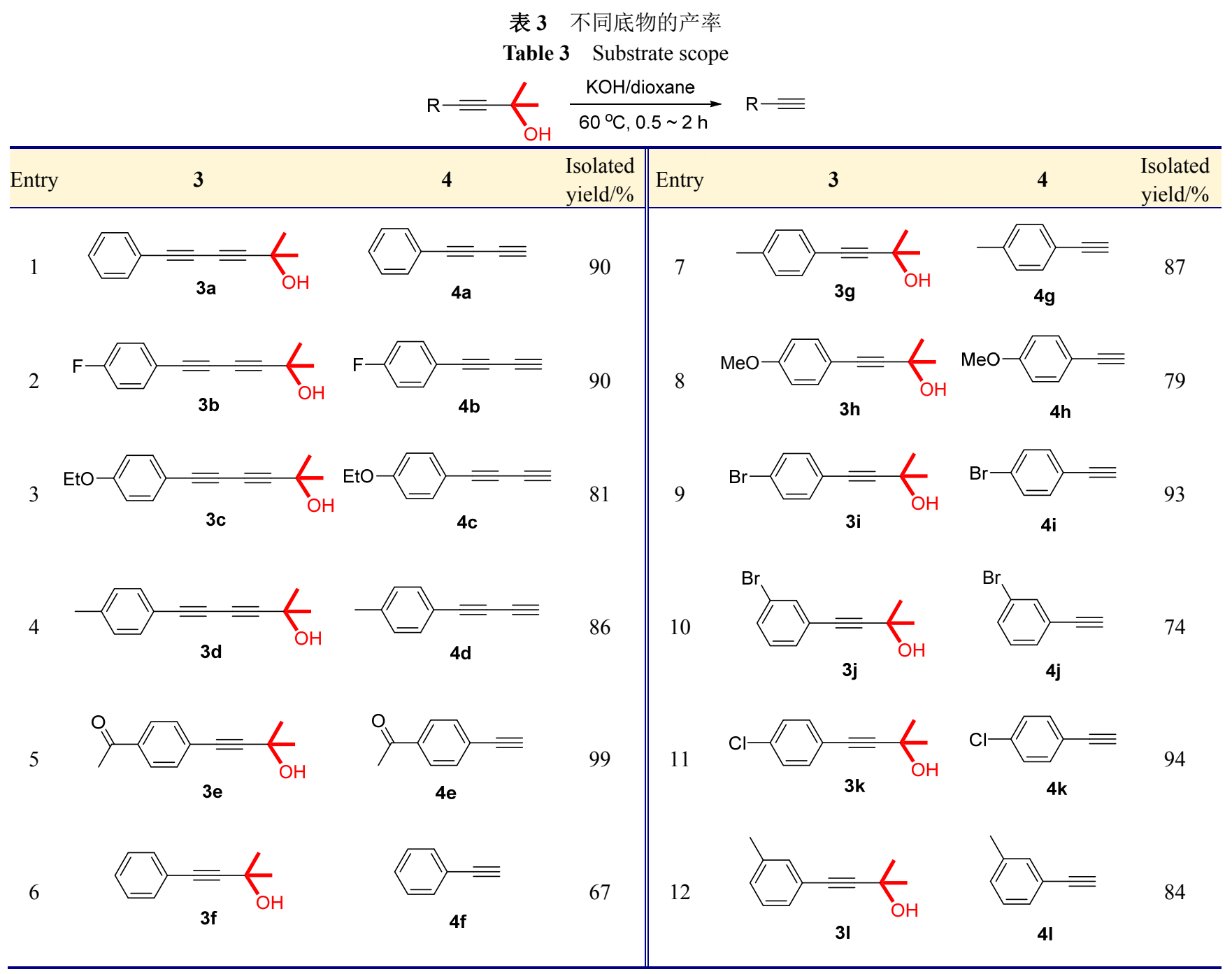


比传统方法 (110 ${ }^{\circ} \mathrm{C}$ 甲苯回流)具备明显的优点, 尤其是 对末端二炔烃的制备, 反应可以在 15 30 min 内完成, 避免了高温下的副反应的发生, 从而完善了制备末端炔 烃的成本相对低廉的方法.

\section{3 实验部分}

\section{1 仪器与试剂}

${ }^{1} \mathrm{H}$ NMR 和 ${ }^{13} \mathrm{C}$ NMR 用 Bruker ADVANCE III 400 $\mathrm{MHz}$ 测定, 以 $\mathrm{TMS}$ 为内标, $\mathrm{CDCl}_{3}$ 为溶剂; 气相色谱在 Agilent 7820A GC System 上测定. 所用试剂均为分析 纯, 甲苯、1,4-二氧六环在氩气氛中金属钠存在下回流 4 $\mathrm{h}$ 后蒸出密封备用. 其余溶剂均以分子篮干燥.

\section{2 实验方法}

取 $0.5 \mathrm{mmol} 3$ 于 $15 \mathrm{~mL}$ 的 Schlenk 管中, 加入磁子、 $\mathrm{KOH}(1.0 \mathrm{mmol})$, 用双排管将体系置换为氩气氛. 保持 氩气正压情况下, 向体系中加入 $2 \mathrm{~mL}$ 干燥过的 1,4-二 氧六环, $60{ }^{\circ} \mathrm{C}$ 油浴中反应 $30 \mathrm{~min}$, 冷却至室温. 向体系 中加入 $4 \mathrm{~mL}$ 水, 以乙酸甲酯进行萃取 $(2 \mathrm{~mL} \times 2)$. 有机 相以无水硫酸钠干燥后, 浓缩, 柱层析. 以正己烷为淋 洗剂, 得到对应的产物 $\mathbf{4}$.

1-苯基-1,3-丁二炔 $(4 a))^{[14]}$ : 淡黄色液体. ${ }^{1} \mathrm{H}$ NMR (300 MHz, $\left.\mathrm{CDCl}_{3}\right) \delta: 7.55$ (d, $\left.J=6.8 \mathrm{~Hz}, 2 \mathrm{H}\right), 7.35 \sim 7.42$ $(\mathrm{m}, 3 \mathrm{H}), 2.52(\mathrm{~s}, 1 \mathrm{H}) ;{ }^{13} \mathrm{C}$ NMR $\left(75 \mathrm{MHz}, \mathrm{CDCl}_{3}\right) \delta$ : 133.0, 129.6, 128.5, 121.0, 75.4, 73.5, 71.3, 68.2.

1-对氟苯基-1,3-丁二炔 $(\mathbf{4 b})^{[14]}$ : 黄色固体. m.p. 69 70 ${ }^{\circ} \mathrm{C} ;{ }^{1} \mathrm{H}$ NMR $\left(300 \mathrm{MHz}, \mathrm{CDCl}_{3}\right) \delta: 7.49 \sim 7.52$ (m, 2H), $7.03(\mathrm{t}, J=8.8 \mathrm{~Hz}, 2 \mathrm{H}), 2.48(\mathrm{~s}, 1 \mathrm{H}) ;{ }^{13} \mathrm{C} \mathrm{NMR}$ $\left(75 \mathrm{MHz}, \mathrm{CDCl}_{3}\right) \delta: 164.5,162.0,134.9,116.0,74.3,73.3$, 71.3, 68.0.

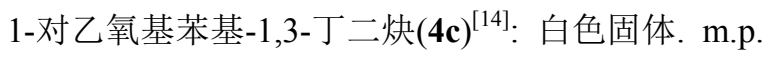
$57 \sim 58{ }^{\circ} \mathrm{C} ;{ }^{1} \mathrm{H}$ NMR $\left(300 \mathrm{MHz}, \mathrm{CDCl}_{3}\right) \delta: 7.44(\mathrm{~d}, J=8.8$ $\mathrm{Hz}, 2 \mathrm{H}), 6.83(\mathrm{~d}, J=9.2 \mathrm{~Hz}, 2 \mathrm{H}), 4.01 \sim 4.06(\mathrm{~m}, 2 \mathrm{H})$, 2.46 (s, $1 \mathrm{H}), 1.42$ (t, $J=7.2 \mathrm{~Hz}, 3 \mathrm{H}) ;{ }^{13} \mathrm{C}$ NMR $(75 \mathrm{MHz}$, $\left.\mathrm{CDCl}_{3}\right) \delta: 160.0,134.4,114.6,112.6,75.7,72.3,70.7$, 68.4, 63.6, 14.7.

1-对甲苯基-1,3-丁二炔 (4d $)^{[14]}$ : 浅黄色液体. ${ }^{1} \mathrm{H}$ NMR (300 MHz, $\left.\mathrm{CDCl}_{3}\right) \delta: 7.40$ (d, $\left.J=8.0 \mathrm{~Hz}, 2 \mathrm{H}\right), 7.13$ (d, $J=8.0 \mathrm{~Hz}, 2 \mathrm{H}), 2.46(\mathrm{~s}, 1 \mathrm{H}), 2.35(\mathrm{~s}, 3 \mathrm{H}) ;{ }^{13} \mathrm{C} \mathrm{NMR}$ $\left(75 \mathrm{MHz}, \mathrm{CDCl}_{3}\right) \delta: 139.2,131.9,128.5,117.2,74.7,72.2$, 70.2, 67.6, 20.8.

4-乙炔基苯乙酮 (4e): 黄色固体. m.p. $69 \sim 70{ }^{\circ} \mathrm{C}$ (文 献值 ${ }^{[24]}$ : 68 70 $\left.{ }^{\circ} \mathrm{C}\right) ;{ }^{1} \mathrm{H}$ NMR (600 MHz, $\left.\mathrm{CDCl}_{3}\right) \delta: 7.92$ (d, $J=8.0 \mathrm{~Hz}, 2 \mathrm{H}), 7.58$ (d, $J=8.0 \mathrm{~Hz}, 2 \mathrm{H}), 3.26(\mathrm{~s}, 1 \mathrm{H})$, $2.61(\mathrm{~s}, 3 \mathrm{H}) ;{ }^{13} \mathrm{C}$ NMR $\left(151 \mathrm{MHz}, \mathrm{CDCl}_{3}\right) \delta: 197.30$, 136.76, 132.30, 128.19, 126.92, 82.75, 80.37, 26.66.
苯乙炔 (4f): 浅黄色液体。 ${ }^{1} \mathrm{H}$ NMR $(600 \mathrm{MHz}$, $\left.\mathrm{CDCl}_{3}\right) \delta: 7.40 \sim 7.55$ (m, 2H), $7.29 \sim 7.39$ (m, 3H), 3.08 $(\mathrm{s}, 1 \mathrm{H}) ;{ }^{13} \mathrm{C}$ NMR $\left(151 \mathrm{MHz}, \mathrm{CDCl}_{3}\right) \delta: 132.10,128.78$, $128.29,122.04,83.63,77.15$.

4-乙炔基甲苯 (4g): 浅黄色液体。 ${ }^{1} \mathrm{H}$ NMR $(600$ $\left.\mathrm{MHz}, \mathrm{CDCl}_{3}\right) \delta: 7.38$ (d, $\left.J=8.0 \mathrm{~Hz}, 2 \mathrm{H}\right), 7.12$ (d, $J=7.9$ $\mathrm{Hz}, 2 \mathrm{H}), 3.03$ (s, 1H), 2.35 (s, 3H); ${ }^{13} \mathrm{C}$ NMR (151 MHz, $\left.\mathrm{CDCl}_{3}\right) \delta: 138.94,132.00,129.05,119.01,83.83,76.45$, 21.49 .

4-乙炔基苯甲醚(4h): 无色液体. ${ }^{1} \mathrm{H}$ NMR (600 $\mathrm{MHz}, \mathrm{CDCl}_{3}$ ) $\delta: 7.43$ (d, $\left.J=8.8 \mathrm{~Hz}, 2 \mathrm{H}\right), 6.84$ (d, $J=8.9$ $\mathrm{Hz}, 2 \mathrm{H}), 3.81$ (s, 3H), 3.00 (s, 1H); ${ }^{13} \mathrm{C}$ NMR $(151 \mathrm{MHz}$, $\left.\mathrm{CDCl}_{3}\right) \delta: 159.92,133.58,114.14,113.92,83.66,75.77$, 55.28 .

1-溴-4-乙炔苯(4i): 黄色固体, m.p. $64 \sim 65{ }^{\circ} \mathrm{C}$ (文献 值 $\left.{ }^{[25]}: 64^{\circ} \mathrm{C}\right) .{ }^{1} \mathrm{H}$ NMR $\left(400 \mathrm{MHz}, \mathrm{CDCl}_{3}\right) \delta: 7.45(\mathrm{~d}, J=$ $8.5 \mathrm{~Hz}, 2 \mathrm{H}), 7.34(\mathrm{~d}, J=8.5 \mathrm{~Hz}, 2 \mathrm{H}), 3.12(\mathrm{~s}, 1 \mathrm{H}) ;{ }^{13} \mathrm{C}$ NMR $\left(101 \mathrm{MHz}, \mathrm{CDCl}_{3}\right) \delta: 133.52,131.57,123.12$, $121.00,82.55,78.36$.

1-溴-3-乙炔苯 $(\mathbf{4 j})$ : 浅黄色液体. ${ }^{1} \mathrm{H}$ NMR (400 $\mathrm{MHz}, \mathrm{CDCl}_{3}$ ) $\delta: 7.63$ (t, $\left.J=1.8 \mathrm{~Hz}, 1 \mathrm{H}\right), 7.48$ (ddd, $J=$ $8.0,2.0,1.1 \mathrm{~Hz}, 1 \mathrm{H}), 7.41(\mathrm{dt}, J=7.8 \mathrm{~Hz}, 1.3 \mathrm{~Hz}, 1 \mathrm{H}), 7.18$ (t, $J=7.9 \mathrm{~Hz}, 1 \mathrm{H}), 3.12(\mathrm{~s}, 1 \mathrm{H}) ;{ }^{13} \mathrm{C}$ NMR $(101 \mathrm{MHz}$, $\left.\mathrm{CDCl}_{3}\right) \delta: 134.83,131.99,130.66,129.74,124.05,122.06$, $82.01,78.55$.

1-氯-4-苯乙炔 $(4 \mathbf{k})$ : 黄色固体. m.p. $47 \sim 48{ }^{\circ} \mathrm{C}$ (文 献值 ${ }^{[26]}$ : $\left.45.4 \sim 46.1{ }^{\circ} \mathrm{C}\right) ;{ }^{1} \mathrm{H}$ NMR $\left(400 \mathrm{MHz}, \mathrm{CDCl}_{3}\right) \delta$ : $7.41(\mathrm{~d}, J=8.5 \mathrm{~Hz}, 2 \mathrm{H}), 7.32 \sim 7.27(\mathrm{~m}, 2 \mathrm{H}), 3.11(\mathrm{~s}, 1 \mathrm{H})$; ${ }^{13} \mathrm{C}$ NMR $\left(101 \mathrm{MHz}, \mathrm{CDCl}_{3}\right) \delta: 134.88,133.34,128.66$, $120.55,82.50,78.18$.

3-甲基苯乙炔(4I): 浅黄色液体. ${ }^{1} \mathrm{H}$ NMR (400 MHz, $\left.\mathrm{CDCl}_{3}\right) \delta: 7.36 \sim 7.26(\mathrm{~m}, 2 \mathrm{H}), 7.23 \sim 7.12(\mathrm{~m}, 2 \mathrm{H}), 3.04$ (s, 1H), $2.32(\mathrm{~s}, 3 \mathrm{H}) ;{ }^{13} \mathrm{C}$ NMR (101 MHz, $\left.\mathrm{CDCl}_{3}\right) \delta$ : 138.00, 132.67, 129.66, 129.18, 128.19, 121.87, 83.82, $76.76,21.18$.

辅助材料(Supporting Information) 所有产物的 ${ }^{1} \mathrm{H}$ $\mathrm{NMR}$ 和 ${ }^{13} \mathrm{C}$ NMR 谱图. 这些材料可以免费从本刊网站 (http://sioc-journal.cn/)上下载.

\section{References}

[1] Siemsen, P.; Livingston, R. C.; Diederich, F. Angew. Chem., Int. Ed. 2000, 39, 2632.

[2] Shun, A. L. K. S.; Tykwinski, R. R. Angew. Chem., Int. Ed. 2006, $45,1034$.

[3] Ross, C.; Scherlach, K.; Kloss, F.; Hertweck, C. Angew. Chem., Int. Ed. 2014, 53, 7794. 
[4] Tykwinski, R. R.; Chalifoux, W.; Eisler, S.; Lucotti, A.; Tommasini, M.; Fazzi, D.; Del, Z. M.; Zerbi, G. Pure Appl. Chem. 2010, 82, 891.

[5] Chalifoux, W. A.; Tykwinski, R. R. Nat. Chem. 2010, 2, 967.

[6] Shi, W. Curr. Organocatal. 2015, 2, 2.

[7] Tykwinski, R. R.; Chalifoux, W.; Eisler, S.; Lucotti, A.; Tommasini, M.; Fazzi, D.; Del Zoppo, M.; Zerbi, G. Pure Appl. Chem. 2010, 82, 891.

[8] Shi, W.; Lei, A. Tetrahedron Lett. 2014, 55, 2763.

[9] Stefko, M.; Tzirakis, M. D.; Breiten, B.; Ebert, M.-O.; Dumele, O.; Schweizer, W. B.; Gisselbrecht, J.-P.; Boudon, C.; Beels, M. T.; Biaggio, I.; Diederich, F. Chem.-Eur. J. 2013, 19, 12693.

[10] Sahnoune, H.; Baranova, Z.; Bhuvanesh, N.; Gladysz, J. A.; Halet, J.-F. Organometallics 2013, 32, 6360.

[11] Bai, D.; Li, C.; Li, J.; Jia, X. Chin. J. Org. Chem. 2012, 32, 994 (in Chinese).

(白东虎, 李春举, 李健, 贾学顺, 有机化学, 2012, 32, 994.)

[12] Wang, Y.-F.; Deng, W.; Liu, L.; Guo, Q.-X. Chin. J. Org. Chem. 2005, 25, 8 (in Chinese) (王晔峰, 邓维, 刘否, 郭庆祥, 有机化学, 2005, 25, 8.)

[13] Shi, W.; Luo, Y. D.; Luo, X. C.; Chao, L.; Zhang, H.; Wang, J.; Lei, A. W. J. Am. Chem. Soc. 2008, 130, 14713.

[14] Zhang, F.-G.; Ma, H.; Zheng, Y.; Ma, J.-A. Tetrahedron 2012, 68, 7663.
[15] Xue, Y.-X.; Zhu, Y.-Y.; Gao, L.-M.; He, X.-Y.; Liu, N.; Zhang, W.-Y.; Yin, J.; Ding, Y.; Zhou, H.; Wu, Z.-Q. J. Am. Chem. Soc. 2014, 136, 4706

[16] Carpita, A.; Mannocci, L.; Rossi, R. Eur. J. Org. Chem. 2005, 1859.

[17] Li, S.; Mao, F.; Shi, Y.; Wang, Y.; Shi, W. J. Fujian Normal Univ. (Nat. Sci.) 2015, 31, 120 (in Chinese).

(李硕, 毛凤, 师瑶, 王阳, 石炜, 福建师范大学学报 (自然科学 版), 2015, 31, 120.)

[18] Jiang, M. X.-W.; Rawat, M.; Wulff, W. D. J. Am. Chem. Soc. 2004, $126,5970$.

[19] Havens, S. J.; Hergenrother, P. M. J. Org. Chem. 1985, 50, 1763.

[20] Danilkina, N. A.; Kulyashova, A. E.; Khlebnikov, A. F.; Bräse, S.; Balova, I. A. J. Org. Chem. 2014, 79, 9018.

[21] West, K.; Wang, C.; Batsanov, A. S.; Bryce, M. R. J. Org. Chem. 2006, 71, 8541 .

[22] Li, J.; Huang, P. Beilstein J. Org. Chem. 2011, 7, 426.

[23] Li, J.; Guo, K.; Dong, Q.; Huang, P.; Wang, H.; Xu, B. CN 104177209, 2014 [Chem. Abstr. 2014, 2020360].

[24] Kabalka, G. W.; Zhou, L.-L.; Wang, L.; Pagni, R. M. Tetrahedron 2006, 62, 857 .

[25] Ghaffarzadeh, M.; Bolourtchian, M.; Fard, Z. H.; Halvagar, M. R.; Mohsenzadeh, F. Synth. Commun. 2006, 36, 1973.

[26] Vaughn, T. H.; Nieuwland, J. A. J. Am. Chem. Soc. 1934, 56, 1207.

(Li, L.; Fan, Y.) 